\title{
Effects of User Age on Smartphone and Tablet Use, Measured with an Eye-tracker via Fixation Duration, Scan-Path Duration, and Saccades Proportion
}

\author{
Suleyman Al-Showarah, Naseer AL-Jawad, and Harin Sellahewa \\ Applied Computing Department, The University of Buckingham, Buckingham, UK \\ \{suleyman.al-showarah; naseer.al-jawad; harin. sellahewa\} @ \\ buckingham.ac.uk
}

\begin{abstract}
The design of user interfaces plays an important role in human computer interaction, especially for smartphones and tablet devices. It is very important to consider the interface design of smartphones for elderly people in order for them to benefit from the variety applications on such devices. The aim of this study is to investigate the effects of user age as well as screen size on smartphone/tablet use. We evaluated the usability of smartphone interfaces for three different age groups: elderly age group $(60+$ years), middle age group (4059 years) and younger age group (20-39 years). The evaluation is performed using three different screen sizes of smartphone and tablet devices: 3.2", 7", and 10.1" respectively. An eye-tracker device was employed to obtain three metrics: fixation duration, scan-path duration, and saccades amplitude. Two hypothesis were considered. First, elderly users will have both local and global processing diffieculties on smartphone/tablet use than other age groups. Second, all user age groups will be influnced by screen sizes; small screen size will have smaller saccades proportion indicating uneasy interface broswing compared to large screen size. All these results have been statistically evaluated using 2-way ANOVA.
\end{abstract}

Keywords: Smartphone interfaces; elderly people; eye tracking; mobile computing; human computer interaction; interfaces evaluation; and usability of smart phone

\section{Introduction}

Smartphones and tablets increasingly play an important role in many aspects of our daily lives. These devices, together with the hundreds of thousands of 'apps' that are available to download onto these devices, are widely used in educational, social, cultural, communication, entertainment, and health related activities. Whilst these devices are commonly owned and used by relatively young users, they could be an integral part of managing the ever increasing ageing population. Therefore, it is important to understand the usability issues of smartphones faced by elderly people and design devices and software applications that are easily accessible and useable by elderly people [4]. 
The importance of research in this area has been highlighted in previous studies such as in [6], [15]. This paper investigates effects of age on smartphone usability.

Few studies have investigated the effects of age on the usability of cell phones [9]. However much has changed in both hardware and applications since these works; changes are introduced at a rapid pace and there is a variety of device manufactures and operating system providers. In order to evaluate user interfaces of smartphones, we need to look at the way the participants interact with these devices. Aspects of interactions to consider includes the way in which users interprets screen components (e.g., icons, widgets, shortcuts, etc.); the way users interact with screen components by gestures (e.g., finger based touch) and by conversational speech [14].

Eye movement analysis has been used in different fields and applications such as in marketing, advertising and user-interface evaluation [3], [19]. Eye movement metrics such as fixation points, durations and scan paths are used widely in studies of Human Computer Interaction (HCI). These metrics give indications related to search efficiency/inefficiency and cognitive processing simple/difficulty [8].

In this paper, we investigated the effects of age and screen size on the usability of smartphones and tablet devices. Essentially, we will examine - based on a number of eye movement metrics -- two hypotheses on the effects of age on smartphone usability. First, previous works have shown that elderly people have difficulties in using technologies [11]. This leads us to predict that elderly users of smartphones and tablet devices will exhibit longer fixation durations (FD) for local processing tasks, and longer scan path durations (SPD) for global processing tasks than users of other age groups (e.g., middle and younger ages). This reflects the cognitive ability of elderly users in browsing content on smartphone interfaces/applications. Second: as the performance task of all age groups will be influnced by screen sizes [7]. Small screen size will have smaller saccades proportion than other screen sizes for complex design of interfaces, espiecally for elderly users.

We used an eye-tracker to record participants' eye movements and fixations during a number of search tasks on three different smartphone/tablet sizes. We will demonstrate that there are significant differences between all age groups in browsing smartphone interfaces. Elderly users will be shown to have less cognitive ability in browsing smartphones interfaces compared to younger age groups.

Also, experiments will show that small screen size will have significantly smaller saccades proportion compared to larger screen sizes of tabelts indicating that the usability in browsing smartphone/tablet interfaces and applications diffiecult for all age groups, more so for elderly users.

The rest of the paper is organized as follows. Related studies on the effects age on technology are discussed in section 2 . Section 3 explains the methodology and procedures relevant to this study, followed by a discussion of experimental results in section 4. Our conclusions and future work are presented in section 5.

\section{$2 \quad$ Related work}

Most existing studies have evaluated the effect of age on PCs. Fukuda et al. conducted a study using an eye tracker to establish the difficulties between elderly people and younger people when browsing web pages on PCs. The results revealed that elderly people have difficulties in using online timetables using PC compared with younger people. The visual perception of elderly people was less efficient than younger age 
group when web browsing. Moreover elderly participants required longer time than the younger ones to accomplish the given tasks [11].

Iwase et al. studied the ability of elderly users to use and acceptance new designs [13]. They conducted the study using a PC mouse for 49 participants that included participants from three different age groups. The results revealed that the mouse pointing time was relatively longer for the elderly group compared with other two age groups, and the error rate increased when distance to the target increases and also when the target size is decreased.

Findlater et al. [10] conducted a study on 20 elderly participants (ages between 61 and 86 years) and 20 adult participants (ages from 19 to 51 years). They used Apple iPads and Apple laptops (Mac OS X 10.7) to examine five tasks: pointing, dragging, crossing, and steering. On the touch screen, they also examined pinch-to-zoom gestures. Results showed that elderly people were slower in using both touch screen and mouse movements in general. Also, the error rate decreased on the touch screen for both age groups. In addition steering was the most difficult task when using a mouse, while dragging was the slowest gesture on the touch screen.

Few studies have evaluated the effect of age on usability of smart/mobile devices. Rogers et al. in [21] conducted a study to evaluate how task demands and user age influenced task performance on touch screen devices and non-touch screen devices (rotary encoder). Their study involved 40 younger (18-28 years) and 40 middle-aged to older adults (i.e. 51-65 years). They used control tasks such as sliders (scrolling), up/down buttons, list boxes, and text boxes. They found that older adults were slower than younger adults on pointing and sliding tasks on a touch screen. Moreover, they found small button sizes were particularly problematic for the older adults. AlShowarah et al. [1] conducted study to examine eye movements of elderly and young participants to find dissimilarities in browsing on different smartphone/tablet applications. Their results found that elderly participants have high dissimilarity than younger ages. In other words, elderly participants were less efficient in browsing smartphone applications/interfaces than younger participants. They also reported that scan paths are stimulus-driven than smartphone screen size driven. Christian et al. [7] conducted a study on three different smartphone display sizes (small: 1.8 inch, medium: 3.6 inch display, large: $7.2 \mathrm{inch}$ ) for 36 participants (average age of younger participants were 26 years, and the average age of elderly participants were: 63 years). The experiment was based on finger gestures on touch screens. The results revealed a clear effect of display size on task performance, where the performance was better on the larger display size. Moreover elderly participants were slower in performing tasks than younger participants.

\section{The Study Methodology and Procedure}

This section describes apparatuses, experiment procedures, stimuli, participants, and dependent variables.

\subsection{Apparatuses}

Eye-tracker. Eyelink-1000 desktop device amounted with IR illuminator was used to track and record eye movements. The illuminator is used to generate reflection patterns on the user's cornea [17], [22]. A chinrest was used to fix both the chin and 
forehead of participants at a distance of 50-55 cm. A distance as recommended from where the participant's eyes will typically is, to the illuminator and eye camera facing the participant. Experiment interfaces (i.e., Smartphone screenshots) were prepared and displayed on the eye-tracker device using Experiment Builder Software. The results of metrics were obtained using Data Viewer [22].

Smartphones. Three mobile devices were used to conduct the experiments: 1) HTC wildfire smartphone, dimensions 106.8 × $60.4 \times 12 \mathrm{~mm}$, screen resolution: $240 \times 320$ pixels, with screen size of 3.2 inches; 2) Samsung Galaxy Tab 2, dimensions 193.7 x $122.4 \times 10.5 \mathrm{~mm}$, screen resolution $1024 \times 600$ pixels, with screen size 7 inches; and 3) Samsung Galaxy Note 10.1 inches, dimensions $262 \times 180 \times 8.9 \mathrm{~mm}$ with a screen resolution of $1280 \times 800$ pixels.

The smartphones/tablets were selected to represent three screen sizes. Small size represents typical smart phones, which have screen sizes between 3 and 5.5 inches. Medium size represents mini tablets, which are typically 7 inches. Large size represents full-size tablet devices which are typically around 10 inches. We chose 3.2 inches to represent small screen sizes, 7 inches to represent medium screen sizes, and 10.1 inches to represent large screen sizes.

\subsection{Participants}

The participants of the experiment were selected from different age groups and they include university students, university staff, and people from the local community. A total of 104 participants participated in the study and they were grouped into three age groups: the elderly group (EG), which consists of 22 participants aged 60 and over; the middle-age group (MG) consisting 31 participants aged 40-59; and the young group (YG), which consists of the remaining 51 participants aged 20-39. In terms of selecting the elderly age group, there are no agreed definitions on the age range in the literature. However, the United Nations considers elderly population to be those $60+$ years of age [18].

\subsection{Experiments Structure}

Each smartphone size has two experiments: EXP1 and EXP2. Each experiment is conducted using participants from three age groups. A participant will be involved in only one smartphone size and in one experiment to avoid any influence on the participant's performance. Fig. 1 illustrates how experiments and different groups of participants are organized. 


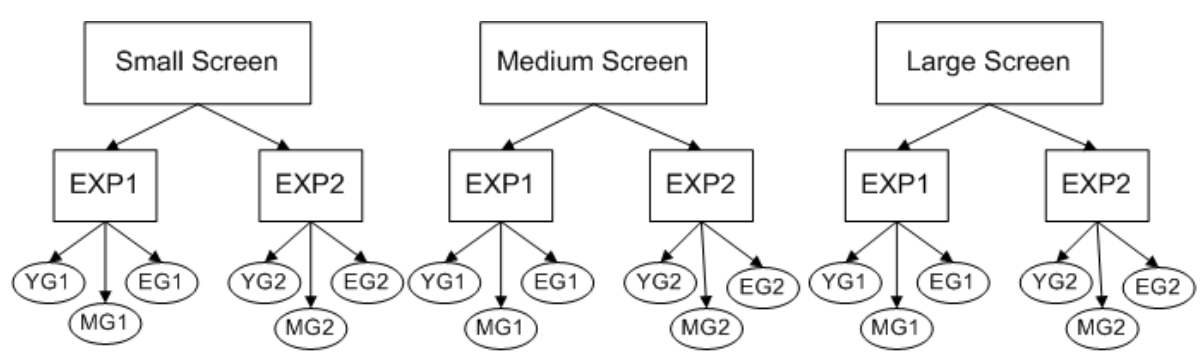

Fig. 1. Organization of smartphone experiments and age groups.

\subsection{Stimuli}

The experiment is composed of nine smartphone applications; each of these applications has two search tasks as shown in Table 1, which forms the two experiment groups (i.e., EXP1 and EXP2) described earlier in Fig. 1. A screenshot of the smartphone application relevant to the search task is displayed on a computer screen that is connected to the eye-tracker.

Table 1. Smartphone applications and questions for experiment one and experiment two.

\begin{tabular}{|c|c|c|c|}
\hline App no & Apps & EXP1 & EXP2 \\
\hline 1 & Skype contact list & $\begin{array}{l}\text { Locate the user who is not } \\
\text { online. }\end{array}$ & Locate the image of David Albert. \\
\hline 2 & $\begin{array}{l}\text { Skype Calling } \\
\text { screen }\end{array}$ & Locate the Backspace button. & Locate the Numbers Field. \\
\hline 3 & $\begin{array}{l}\text { Skype Profile } \\
\text { screen }\end{array}$ & $\begin{array}{l}\text { Locate the button used to change } \\
\text { your current status to be visible }\end{array}$ & $\begin{array}{l}\text { Locate the account holder's Pic- } \\
\text { ture. }\end{array}$ \\
\hline 4 & $\begin{array}{l}\text { Facebook account } \\
\text { holder profile }\end{array}$ & $\begin{array}{l}\text { Locate the number of incoming } \\
\text { messages }\end{array}$ & Locate the account holder's name? \\
\hline 5 & $\begin{array}{l}\text { Yahoo Email } \\
\text { folder list }\end{array}$ & $\begin{array}{l}\text { Locate the number of deleted } \\
\text { messages. }\end{array}$ & $\begin{array}{l}\text { Locate the number of new mes- } \\
\text { sages? }\end{array}$ \\
\hline 6 & Gallery screen & Locate the delete image button? & Locate the Share photo button? \\
\hline 7 & Alarm screen & Locate the active alarm? & $\begin{array}{l}\text { Locate the button to add a new } \\
\text { alarm? }\end{array}$ \\
\hline 8 & $\begin{array}{l}\text { Skype Main } \\
\text { Screen }\end{array}$ & $\begin{array}{l}\text { Locate the button that will show } \\
\text { a list of contacts? }\end{array}$ & Locate the 'exit' button? \\
\hline 9 & Settings screen & $\begin{array}{l}\text { Locate the button to view Wire- } \\
\text { less and networks settings? }\end{array}$ & $\begin{array}{l}\text { Locate the button that lets you } \\
\text { change Sound settings. }\end{array}$ \\
\hline
\end{tabular}

\subsection{Experimental Procedure}

Participants were asked to perform the search tasks described in either EXP1 or EXP2 based on the Smartphone application interfaces of a selected screen-size. Each search task was designed carefully and has a specific answer; this is based on Broder's recommendation in terms of finding specific information on a web page [5]. Each participant was tested individually in a lab environment set for conducting the experiment. 
The aim of the study and a description of the interface contents of the Smartphone applications used for the experiment was given to each participant. This was followed by eye-tracker calibration before starting the experiment.

During an experiment, each participant was asked to find the targets on the presented interfaces based on the nine search tasks. A search task ends when the participant indicates the target for the current search task was found, or if the participant could not find the target within 30 seconds. The next search task is presented after the current one ends.

\subsection{Eye-tracking Metrics}

We used the following four eye-tracking metrics as dependent variables to explore the determinants of visual behavior on smart device interface: 1) Fixation Duration; 2) Saccade Amplitude; 3) Scan-Path Duration; and 4) Saccades Proportion.

Fixation Duration (FD): measures the amount of time the eyes are focused on a particular point on the screen (i.e., local process) [8]. A longer fixation duration reflects the participant's difficulties in locating a given target in the local process [2], [11-12] [20]. FD is measured in milliseconds.

Saccade Amplitude (SA): saccades occur when the eye moves from one fixation to the next fixation [8], [20]. Large length of saccade amplitude gives more meaningful cues and less task difficulty [12]. SA is measured in terms of visual angle degree.

Scan-Path Duration (SPD): a scan-path is a sequence of all fixations and saccades across a visual display [8], [20]. SPD measures global processing of interfaces, where longer SPD indicates less efficient scanning and browsing [2], [12], [17]. SPD is measured in milliseconds.

Saccades Proportion (SP): is a measurement derived from the metrics above - SP is the total length of SA divided by SPD. A larger SP indicates more meaningful search and efficient browsing. Saccades proportion is used to test the effects of screen sizes on browsing efficiency and application usability for all age groups.

Fig. 2 displays an example of FD, SPD, and SA metrics as produced by the eyetracker software tool. 


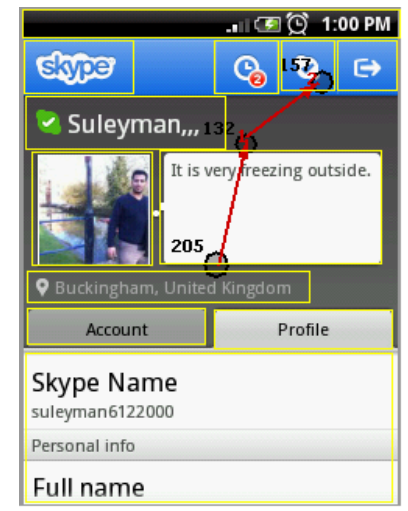

Fig. 2. Visual output of eye-tracking metrics. The circles are tagged by its fixation time in milliseconds, saccades amplitude is represented by the arrows between fixations.

We calculated the average FD, SPA, SPD and SA of each participant (based on nonerroneous search tasks of the 9 search tasks) [7]. A two-way ANOVA test was used to provide us with the average metrics for each age group with a Standard Deviation (STD), and Alpha $(\alpha)$. In ANOVA, we used $\alpha<0.05$, which indicates the confidence level between the tested means, and gives strong evidence against null hypotheses.

\section{$4 \quad$ Experimental Results and Discussions}

We evaluated age groups' effect on each of the three screen sizes using fixation duration and scan-path duration data. Moreover, saccades proportion data were used to find the influence of screen size on all three age groups.

\subsection{Fixation Duration (FD) Results}

Average fixation durations for all three screen sizes are shown in Table 2 and Table 3. The elderly age group took $2922.47 \mathrm{~ms}$ on average (across all screen sizes) to complete an experiment, whilst it took $2375.94 \mathrm{~ms}$ and $1686.45 \mathrm{~ms}$ respectively for the medium and young age groups. The results show a significantly longer fixation duration for elderly participants when compared to younger participants (i.e. $\alpha=0.000$ ), and middle age participants (i.e. $\alpha=0.019$ ). Moreover, FD of the middle-age group is significantly longer than that of the younger-age group. These results support our first hypothesis; elderly users will exhibit difficulties in using smartphones. Also, longer FDs of the EG indicates their difficulties in local processing.

Fig. 3, Fig. 4, and Fig. 5 show the results of the two experiments for small, medium and large screen sizes respectively and they demonstrate effects of age on smartphone usability measured in terms of FD. Our findings are in line with previous works on the effects of age on technology where it has been shown that in general, elderly people are less efficient when using technology [7], [10], [11], [13], and [21]. 


\subsection{Scan-Path Duration (SPD) Results}

Scan-path duration results for all three screen sizes are shown in Table 2, and Table 3. Fig. 3, Fig. 4, and Fig. 5 show results for each screen size. As expected, the elderly group have significantly longer SPD compared to the younger age group for experiments on each of all three screen sizes. Moreover, the middle-age group has a significantly longer SPD than the younger age group. However, there is no significant difference between SPDs of elderly and middle age groups (i.e. $\alpha=0.182$ ).

Scan-path duration is used as a metric to discover if elderly users find difficulties in processing global information. The results of small, medium and large screen sizes demonstrate that elderly users find it difficult to process global information on smartphone interfaces.

These results support our first hypothesis; elderly users will exhibit difficulties in processing local and global information on smartphone applications. These results concur with previous works as highlighted in Sec. 4.1.
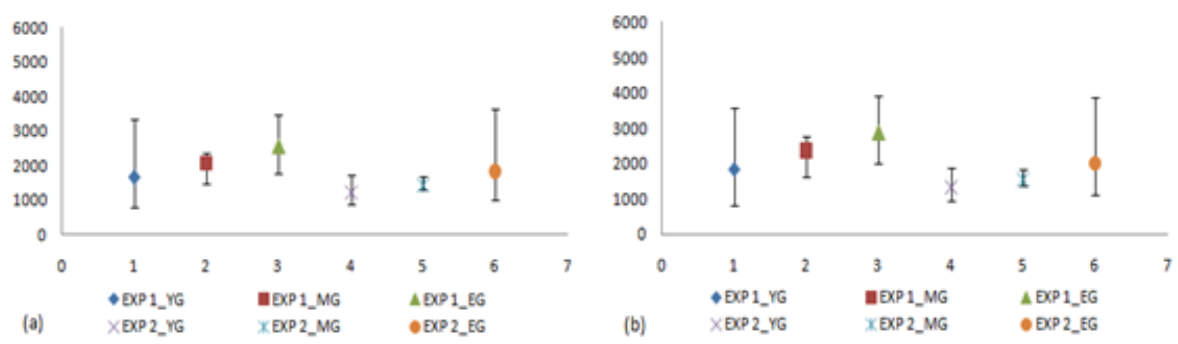

Fig. 3. Eye-tracking metrics for small screen size. (a) Mean FD, and (b) Mean SPD, in millisecond.
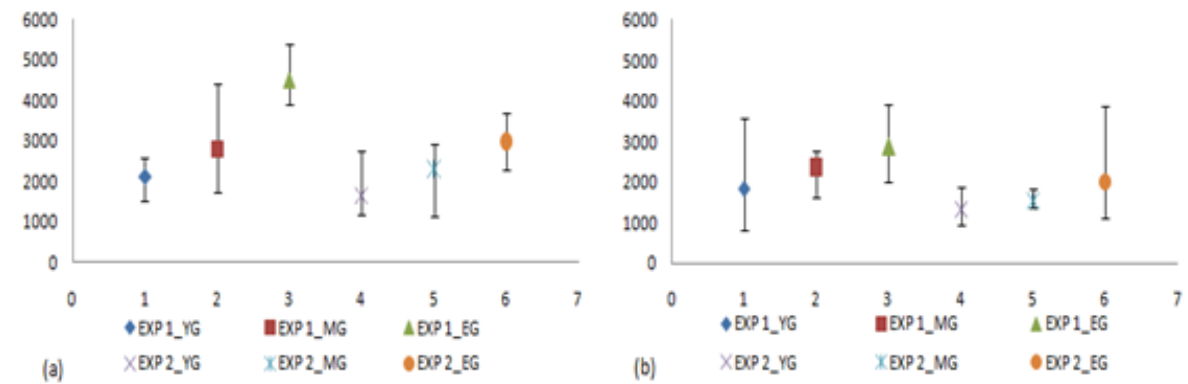

Fig. 4. Eye-tracking metrics for medium screen size. (a) Mean FD, and (b) Mean SPD, in millisecond 

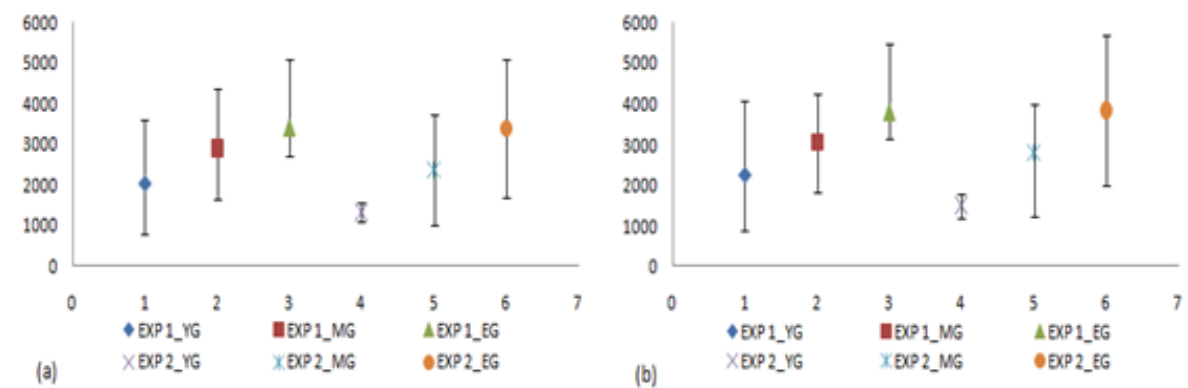

Fig. 5. Eye-tracking metrics for large screen size. (a) Mean FD, and (b) Mean SPD, in millisecond.

Table 2. Mean and STD of FDs and SPDs across all three screen sizes for each age group when $\alpha<0.05$.

\begin{tabular}{lllllll}
\hline $\begin{array}{l}\text { Age } \\
\text { Metrics }\end{array}$ & Group/ & YG & \multicolumn{3}{c}{ MG } & \multicolumn{2}{c}{ EG } \\
\hline FD & mean & STD & mean & STD & mean & STD \\
SPD & $1686 \downarrow$ & 701.47 & 2376 & 857.84 & $2922 \uparrow$ & 1267.19 \\
\hline
\end{tabular}

Table 3. Means all metrics: Fixation Durations, Scan-Path Durations.

\begin{tabular}{|c|c|c|c|c|c|c|c|c|c|c|c|c|}
\hline \multirow{3}{*}{$\begin{array}{l}\text { Zे } \\
0 \\
0 \\
0 \\
0 \\
0\end{array}$} & \multicolumn{4}{|c|}{ Small Screen } & \multicolumn{4}{|c|}{ Medium Screen } & \multicolumn{4}{|c|}{ Large Screen } \\
\hline & \multicolumn{2}{|c|}{ FD } & \multicolumn{2}{|c|}{ SPD } & \multicolumn{2}{|c|}{ FD } & \multicolumn{2}{|c|}{ SPD } & \multicolumn{2}{|r|}{ FD } & \multicolumn{2}{|c|}{ SPD } \\
\hline & $\begin{array}{l}\text { Фี } \\
\text { }\end{array}$ & $\stackrel{\rho}{\mathrm{s}}$ & $\begin{array}{l}\text { च } \\
\stackrel{\Xi}{\Xi}\end{array}$ & $\stackrel{\theta}{\theta}$ & $\begin{array}{l}\text { च } \\
\stackrel{\Xi}{\Xi}\end{array}$ & $\stackrel{\theta}{\theta}$ & 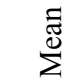 & $\underset{n}{\ominus}$ & 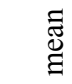 & $\stackrel{\theta}{\theta}$ & $\begin{array}{l}\text { चี } \\
\stackrel{\Xi}{\Xi}\end{array}$ & $\underset{n}{\theta}$ \\
\hline EG & 2067 & 922 & 2152 & 1020 & 3615 & 1009 & 3401 & 1404 & 3397 & 1380 & 3812 & 1460 \\
\hline & $\uparrow$ & & & & $\uparrow$ & & $\uparrow$ & & $\uparrow$ & & $\uparrow$ & \\
\hline MG & 1825 & 458 & 2325 & 768 & 2542 & 770 & 3142 & 937 & 2607 & 1096 & 2927 & 1097 \\
\hline YG & 1467 & 701 & 1604 & 710 & 1891 & 569 & 2167 & 686 & 1738 & 777 & 1949 & 851 \\
\hline & $\downarrow$ & & & & $\downarrow$ & & $\downarrow$ & & $\downarrow$ & & $\downarrow$ & \\
\hline
\end{tabular}

\subsection{Effect of Screen Size on Smartphone Usability}

The results in Table 4 and Table 5 show the effect of smartphone screen size, measured by saccades proportion, on three age groups. Note that larger values of SPs indicates better usability of smartphone interfaces, easier browsing and more meaningful cues [12]. As expected in our second hypothesis, screen size has had an effect on the usability of smartphones by users of all age groups, especially elderly users.

The average SP on small screens is smaller than medium and large screen sizes. There is no significant difference between the SPs of small and medium screen sizes 
(i.e., $\alpha=0.981$ ). But there is a significant difference between the SPs of small and large screen sizes, and between medium and large screen sizes (i.e., $\alpha=0.000$ ). These observations indicate that large screen size has had a better effect on the usability of smartphones.

However the average experience of participant in using smartphones who were involved in small screen size was larger than middle and large screen sizes; the small screen size still has smaller saccades proportion for difficult use. The average experience in small, medium, and large screen sizes are: (1.14 years), (0.82 years) and (0.76 years) respectively as shown in Fig. 6.

To the best of our knowledge, we have not come across a research study conducted to analyze the effects of smartphone screen size on users of different age groups elderly users based on saccades proportion captured using an eye tracker.

The average SP for elderly (across all screen sizes) is smaller than middle and younger age groups. There is a significant difference between the SPs of younger and middle ages (i.e., $\alpha=0.000$ ). Similarly, there is a significant difference between the SPs of younger ages and elderly ages, and between middle ages and elderly ages (i.e., $\alpha=0.008)$. This further indicates that users in the elderly age group find smartphones/tablets less useable than their counterparts in younger age groups.

When compared with other studies that used different tools and metrics, our findings are in line with works such as in [7] that elderly users were influnced in their performance to be less on small screen size than larger screen sizes

Table 4. Mean saccades propotions of three screen sizes. significants differences for means of screen sizes when $(\alpha<0.05)$

\begin{tabular}{lllllll}
\hline \multirow{2}{*}{ Metrics/Screen Sizes } & Small & \multicolumn{3}{c}{ Medium } & Large \\
& Mean & STD & Mean & STD & mean & STD \\
\hline Saccades Proportion & $1.219 \downarrow$ & 0.303 & 1.221 & 0.395 & $1.584 \uparrow$ & 0.669 \\
\hline
\end{tabular}

Table 5. Mean of saccades proportion forl three age groups. Significant differences between means of age groups are $(\alpha<0.05)$.

\begin{tabular}{lllllll}
\hline Age Group & YG & & MG & & EG \\
mean & STD & mean & STD & mean & STD \\
\hline Saccades Proportion & $1.65 \uparrow$ & 0.493 & 1.15 & 0.217 & $0.893 \downarrow$ & 0.305 \\
\hline
\end{tabular}




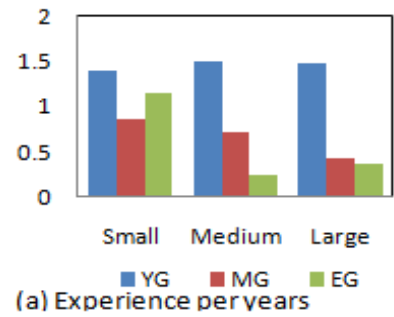

(a) Experience pervears

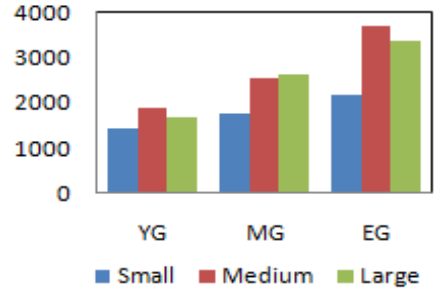

(b) Millesconds

Fig. 6. (a) Participants' experience in using smartphone/tablets (b) Mean FD for three age groups and three smartphone screen sizes.

\section{Conclusions and Future work}

In this study we analyzed the effects of age on smartphone and tablet use. Also, we investigated if screen size has an effect on smartphone/tablet usability. Experiments were conducted on three screen sizes using participants of three different age groups. An eye-tracker was used to measure fixation, scan paths and saccades.

Elderly people were less efficient and have less cognitive ability in browsing smartphone interfaces. They exhibited more difficulties in processing information at both local and global level on smartphones across all screen sizes than middle and younger age groups. The results of saccades proportion indicated that the usability in browsing smartphone/tablet interfaces and applications on a small screen size is difficult for all age groups - more so for elderly user group - compared to larger (i.e., tablet size) screen sizes.

In general, the results revealed a possible relationship between getting older with less experience in using smartphones and the complexity of interface design with smaller screen sizes of smartphone for elderly users.

In future work, we will analyze the influence of age and screen size on the accuracy and efficiency of gestures on smartphones.

Acknowledgments. The authors would like to thank Dr David Windridge from University of Surrey, UK, for lending the eye tracker machine to collect data for this study, and all participants of the experiments. Also, they would like to thank the University of Buckingham's PGRCF grant for part sponsoring this research.

\section{References}

1. Al-Showarah, S., Al-Jawad, N., Sellahewa, H., 2013. Examining eye-tracker scan paths for elderly people using smart phones, York Doctoral Symbosium (YDS), the paper has been published as a York Computer Science technical report. University of York, U K.

2. Al-Wabil, A., 2009. The Effect of Dyslexia on Web Navigation, City University London, UK: s.n. 
3. Andrienko, G., Andrienko, N., Burch, M. \& Weiskopf, D., 2012. Visual Analytics Methodology for Eye Movement Studies. Visualization and Computer Graphics, IEEE Transactions on, 18(12), pp. 2889-2898.

4. Balakrishnan, S., Salim, S. \& Hong, J. L., 2012. User Centered Design Approach for Elderly People in Using Website. s.1., s.n., pp. 382-387.

5. Broder, A., 2002. A taxonomy of web search. s.1., s.n., pp. 3-10.

6. Caprani, N., O'Connor, N. E. \& Gurrin, C., 2012. Touch screens for the older user.

7. Christian, Wandke, H. \& Blessing, L., 2010. Gestural interfaces for elderly users: help or hindrance?. In: Gesture in Embodied Communication and Human-Computer Interaction. s.l.:Springer, pp. 269-280.

8. Cooke, L., 2006. Is Eye Tracking the Next Step in Usability Testing?. 0-7803-97789/06/\$20.00 C2006 IEEE., p. 7.

9. Dongfang, Z. \& Qiang, S., 2009. A discussion based on a design of cell phones usability by the elderly in China. s.l., s.n., pp. 1385-1389.

10. Findlater, L. et al., 2013. Age-related differences in performance with touchscreens compared to traditional mouse input. s.l., s.n., pp. 343-346.

11. Fukuda, R. \& Bubb, H., 2010. Eye tracking study on Web-use: Comparison between younger and elderly users in case of search task with electronic timetable service. December.p. 27.

12. Goldberg, J. H. et al., 2002. Eye tracking in web search tasks: design implications. s.1., s.n., pp. 51-58.

13. Iwase, H. \& Murata, A., 2002. Comparison of mouse performance between young and elderly - basic study for designing mouse proper for elderly. s.1., s.n., pp. 246-251.

14. Jacob, R. J., 2006. Eye Tracking in Advanced Interface Design. p. 53.

15. Kim, H. et al., 2007. Contextual Research on Elderly Usersâ€ $€^{\mathrm{TM}}$ Needs for Developing Universal Design Mobile Phone. Volume 4554, pp. 950-959.

16. Miyoshi, T. \& Murata, A., 2001. Usability of input device using eye tracker on button size, distance between targets and direction of movement. s.l., s.n., pp. 227-232 vol.1.

17. Nettleton, D. \& Gonzalez-Caro, C., 2012. Analysis of User Behavior for Web Search Success Using Eye Tracker Data. s.1., s.n., pp. 57-63.

18. Organization, W. H. \& others, 2010. Definition of an older or elderly person. Health statistics and health information systems http://www. who. int/healthinfo/survey/ageingdefnolder/en/index. html (accessed 21 June 2012).

19. Poole, A. \& Ball, L. J., 2004. Eye Tracking in Human-Computer Interaction and Usability Research: Current Status and Future Prospects.

20. Rayner, K., 1998. Eye movements in reading and information processing: 20 years of research. Psychological bulletin, 124(3), p. 372.

21. Rogers, W. A., Fisk, A. D., McLaughlin, A. C. \& Pak, R., 2005. Touch a screen or turn a knob: Choosing the best device for the job. Human Factors: The Journal of the Human Factors and Ergonomics Society, 47(2), pp. 271-288.

22. SR_Research.Ltd, 2010. EyeLink? 1000 Installation Guide Tower, Desktop, LCD Arm, Primate, and Long Range Mounts Remote, $2000 \mathrm{~Hz}$ and Fiber Optic Camera Upgrades, s.1.: s.n. 